\title{
One-year effect of wearing orthokeratology lenses on the visual quality of juvenile myopia: a retrospective study
}

\author{
Yewei Yin ${ }^{1}$, Yang Zhao ${ }^{1}$, Xiaoying Wu ${ }^{1}$, Mengyang Jiang ${ }^{1}$, Xiaobo Xia ${ }^{1}$, Yao Chen ${ }^{1}$, Weitao Song ${ }^{1}$, Shengfa Hu ${ }^{1}$, \\ Xia Zhou ${ }^{1}$, Kelly Young ${ }^{2}$, Dan Wen ${ }^{\text {Corresp. } 1}$ \\ ${ }^{1}$ Department of Ophthalmology, XiangYa Hospital, Central South University, Changsha, China \\ 2 Department of Veterans Affairs, Miami, United States of America \\ Corresponding Author: Dan Wen \\ Email address: 912096313@qq.com
}

Objective. To study the one-year effect of wearing orthokeratology (OK) lenses on the visual quality of juvenile myopia. Methods. The right eyes of 36 juvenile myopias were retrospectively studied in this work. Q-value, e-value, corneal curvature, strehl ratio (SR), modulation transfer function (MTF) and wavefront aberration (WA) were compared before and at 1, 3 and 12 months after wearing OK lenses. The SR, MTF and WA of cornea, internal optic and ocular were analyzed separately. The spherical and cylinder diopter, vision acuity, compensating factor (CF) and compensative rate (CF\%) were compared before and at 12 months after wearing OK lenses. Results. (1) The vision of LogMAR increased and the corneal curvature decreased significantly after wearing OK lenses. There was no significant difference for the e-value before and after wearing OK lenses. The Qvalue increased at 1 month but decreased at 3 and 12 months remarkably. (2) The ocular and internal optic SR and MTF increased significantly at 1 month and then remained stable. The MTF in different spacial frequencies increased after wearing OK lenses. There was no significant difference for the corneal SR before and after wearing OK lenses, and the corneal MTF decreased significantly after wearing OK lenses. (3) For the ocular, the total higher order aberration (HOA), spherical, coma and trefoil aberrations increased, and the total aberration, total lower order aberration (LOA) and defocus aberration decreased obviously except astigmatism. The corneal aberrations increased significantly after wearing OK lenses except astigmatism. For the internal optic, the total aberration, total LOA and defocus aberration decreased, and the total HOA, coma and trefoil aberration increased significantly except the astigmatism and spherical aberrations. (4) The CF and CF\% of total aberration, total LOA, total HOA and coma aberrations increased, and those of astigmatism and spherical decreased at 12 months. Conclusions. Orthokeratology is effective in correcting the refractive error and improving the vision quality of juvenile myopia over the one-year follow-up period. 


\section{One-Year Effect of Wearing Orthokeratology Lenses}

2

3

4 Yewei Yin ${ }^{1}$, Yang Zhao ${ }^{1}$, Xiaoying $\mathrm{Wu}^{1}$, Mengyang Jiang ${ }^{1}$, Xiaobo $\mathrm{Xia}^{1}$, Yao Chen ${ }^{1}$, Weitao

5 Song ${ }^{1}$, Shengfa Hu${ }^{1}$, Xia Zhou ${ }^{1}$, Kelly Young ${ }^{2}$, Dan Wen ${ }^{1 *}$

$6 \quad{ }^{1}$ Department of Ophthalmology, XiangYa Hospital, Central South University, Changsha,

7 410008, Hunan, China.

$8 \quad{ }^{2}$ Department of Veterans Affairs, Miami, United States of America

$9 *$ Corresponding author:

10 Dan Wen

11 Department of Ophthalmology, XiangYa Hospital, Central South University, Changsha, 410008,

12 Hunan, China

13 Email address: wendan@csu.edu.cn (DW)

\section{Retrospective Study}

15

16

17

\section{Abstract}

Objective. To study the one-year effect of wearing orthokeratology (OK) lenses on the visual quality of juvenile myopia.

Methods. The right eyes of 36 juvenile myopias were retrospectively studied in this work. Qvalue, e-value, corneal curvature, strehl ratio (SR), modulation transfer function (MTF) and wavefront aberration (WA) were compared before and at 1, 3 and 12 months after wearing OK lenses. The SR, MTF and WA of cornea, internal optic and ocular were analyzed separately. The spherical and cylinder diopter, vision acuity, compensating factor (CF) and compensative rate (CF\%) were compared before and at 12 months after wearing OK lenses.

4 Results. (1) The vision of LogMAR increased and the corneal curvature decreased significantly after wearing OK lenses. There was no significant difference for the e-value before and after wearing OK lenses. The Q-value increased at 1 month but decreased at 3 and 12 
27 months remarkably. (2) The ocular and internal optic SR and MTF increased significantly at 1 28 month and then remained stable. The MTF in different spacial frequencies increased after

29

30

31 wearing OK lenses. There was no significant difference for the corneal SR before and after wearing OK lenses, and the corneal MTF decreased significantly after wearing OK lenses. (3) For the ocular, the total higher order aberration (HOA), spherical, coma and trefoil aberrations increased, and the total aberration, total lower order aberration (LOA) and defocus aberration decreased obviously except astigmatism. The corneal aberrations increased significantly after wearing OK lenses except astigmatism. For the internal optic, the total aberration, total LOA and defocus aberration decreased, and the total HOA, coma and trefoil aberration increased significantly except the astigmatism and spherical aberrations. (4) The CF and CF\% of total aberration, total LOA, total HOA and coma aberrations increased, and those of astigmatism and spherical decreased at 12 months.

Conclusions. Orthokeratology is effective in correcting the refractive error and improving the vision quality of juvenile myopia over the one-year follow-up period.

\section{Introduction}

Orthokeratology $(\mathrm{OK})$ is a non-invasive technology for refractive correction [1] and has been widely applied to treat young myopic patients since the 1990s [2]. It has been generally recognized that wearing this rigid contact lens at night can effectively reduce myopic degree [3, 4]. With a reverse geometry design, the rigid contact lens is able to flatten the center of the front corneal surface by the pressure induced from the tear film and lids [5]. Meanwhile, the lens plays an important role in controlling myopia progression with a mechanism of myopia shift [6, 7]. Moreover, the orthokeratic effect is reversible, and the cornea can recover to its original shape after stopping wearing the lens for 1 to 6 months $[8,9]$.

In general, most OK users could obtain an effective refractive correction in the daytime. However, there might be some side effects such as the night vision loss, ghosting, halos and glares, particularly within the first month after wearing the lens [10]. Academically, there is still no consensus that wearing OK lenses can help improve the visual quality [11, 12]. The divergence might be associated with the amount of corrected refractive error, lens decentration and different evaluation methods, etc $[13,14]$. In this work, the iTrace aberrometer was used to 
57 evaluate the visual quality objectively, which includes a few parameters that are with good 58 reproducibility, i.e. the asphericity of cornea (Q-value), eccentricity of cornea (e-value), strehl 59 ratio (SR), modulation transfer function (MTF) and wavefront aberration (WA) [15, 16]. 60 Moreover, it is available to record the visual parameters respectively according to cornea, 61 internal optic and ocular, which makes it possible to quantificationally assess the 62 therapeutic effect of orthokeratology.

63 The main purpose of this work is to analyze the one-year effect of wearing OK lenses on the 64 visual quality of juvenile myopia, and explore the possible reasons for the limited alleviation of 65 vision quality. Corresponding statistical results could provide objective evidences to the 66 improvement of visual quality after wearing OK lenses, and make benefits to the fitting of OK 67 lens directly.

\section{Patients and methods}

\subsection{Study subjects and Methods}

36 myopic teenagers were enrolled, and the parameters of their right eyes were analyzed retrospectively. The inclusion criteria was as follows: the age was 6 18 years old, and the spherical diopter was $-1.00 \sim-7.00 \mathrm{D}$, and the cylinder diopter was $0 \mathrm{D} \sim-2.00 \mathrm{D}$, and the treatment duration was more than one year with regular follow-up. The patients with any ocular surface diseases, systemic diseases or a history of wearing any contact lens before the therapy were excluded, and the teenagers who suffered any vision-threatening side events during the therapy were also excluded. The enrolled patients wore the OK lens no fewer than 8 hours per night and removed the lens before 7 am on the next day. The follow-up examinations were performed between 8 and 10 am at each visit. The therapeutic process lasted for more than 1 year from July 1st, 2015 to June 31st, 2017 in the vision care clinic of Xiangya Hospital, Central South University, China. All the subjects underwent the examinations including slitlamp microscope, manifest refraction and iTrace aberration examination before and at 1, 3 and 12 months after wearing OK lenses.

The spherical and cylinder diopter were measured by manifest refraction. The vision acuity was obtained using the standard logarithmic visual acuity charts. The slitlamp microscopy was 
87 by the iTrace aberration analyzer (TRACEY VFA2 iTrace) when the pupil size was $4 \mathrm{~mm}$. All 88 the measurements were repeated 3 times for each eye. The compensating factor (CF) and 89 compensative rate $(\mathrm{CF} \%)$ were obtained from the WAs. $\mathrm{CF}$ was calculated by 1 minus the

90 absolute ratio of ocular WA to corneal WA, and CF\% referred to the ratio of positive CFs to all 91 CFs. The spherical and cylinder diopter, the visual acuity, the $\mathrm{CF}$ and $\mathrm{CF} \%$ were compared 92 before and at 12 months, and the Q-value, e-value, corneal curvature, SR, MTF and WA were 93 analyzed before and at 1, 3 and 12 months. The subjects' personal information was anonymized 94 before the assessment.

95 This research project was reviewed and approved by the Ethic Committee of Xiangya 96 Hospital of Central South University (the IRB approval number is 201505044). The design and 97 methods of this research were in accordance with the requirements of regulations and procedures 98 regarding to the human subject protection laws such as GCP and ICH-GCP. This study adhered 99 to the tenets of the Declaration of Helsinki. Before wearing OK lenses, informed consents were 100 obtained from the subjects and their guardians who fully understood the protocol and potential risks of the OK treatment. Permissions were also acquired to take the medical records of each subject as experimental data for the research purpose.

\subsection{Orthokeratology lens and lens fitting}

105

106

107

108

109

110

111

112

The reverse-geometry OK lens (Japan alpha-ortho K) was applied in this work. The lens was manufactured with Boston EM material, with the central thickness of $0.22 \mathrm{~mm}$, refractive rate of 1.422 and gas permeability of $104 \times 10-11\left(\mathrm{~cm}^{2} \times \mathrm{mlO}_{2}\right) /(\mathrm{s} \times \mathrm{mL} \times \mathrm{mmHg})$. According to the simulated keratometry, corneal curvature and desired refractive change, the subjects were fitted with the lens using the lens-fitting software supplied by the manufacturers. The subjects wore the selected lens for one-hour trial.

\subsection{Statistical Analysis}

The IBM SPSS23.0 statistical packages and Microsoft Excel were used for the data management and analysis. The paired sample t-test was used to analyze the visual acuity, diopter 
115 and CF when the data had a normal distribution, and the Wilcoxon signed rank test was 116 considered when the data distribution was abnormal. The one-way repeated measurement 117 analysis of variance was applied to analyze the differences of the corneal curvature, Q-value, e118 value, SR, MTF and WA before and at 1, 3 and 12 months after wearing OK lenses when the 119 data followed the law of normal distribution; if not, Friedman test was used. $\mathrm{p}<0.05$ was 120 considered statistically significant for the visual acuity, diopter and CF, and the adjusted alpha i.e. $121 \mathrm{alpha} /$ no. of test, was applied for the analysis with repeated measurement.

122

\section{3. Results}

124

125

126

127

128

129

130

131

132

133

134

135

136

137

138

139

140

141

\subsection{Descriptive statistics of the subjects}

The descriptive statistics of the myopic teenagers before and after wearing OK lenses were summarized in Table 1. 19 females and 17 males aged of $(10.8 \pm 2.2)$ years old were enrolled in the study. It was informed that the spherical diopter decreased dramatically from $(-3.403 \pm 1.580)$ to $(-0.410 \pm 0.330)$ at 12 months after wearing OK lenses; whereas the evolution of the cylinder diopter was not obvious, relatively speaking. Moreover, the vision of LogMAR in the daytime increased significantly at 12 months. Two subjects complained about ghosting in the initial period of wearing OK lenses due to severe lens decentration, and this symptom gradually vanished after refitting the lens. Three subjects had night vision impairment in the late period, and a new pair of OK lenses was replaced after one year.

\subsection{Comparison of corneal curvature, Q-value and e-value before and}

\section{after wearing OK lenses}

Since the improvement of vision acuity originates from the reshaping of cornea, the corneal topography analysis is necessary for evaluating the effect of OK treatment. As shown in Table 2, under the pressure induced by the $\mathrm{OK}$ lens, both horizontal and vertical corneal curvatures reduced obviously after 1 month and kept stable subsequently. Meanwhile, Q-values increased at 1 month and gradually reduced at 3 and 12 months. For the e-value, no significant differences 
142 were noticed at 1, 3 and 12 months when compared respectively with that before wearing OK 143 lenses.

144

145 3.3. Comparison of SR and MTF before and after wearing OK lenses

146 The objective evaluation of visual quality is necessary for the correction of refractive errors, 147 and SR and MTF quantitatively represent the variation of vision quality. As summarized in Table 148 3, both the ocular and internal optic SR and MTF increased significantly at 1 month and kept 149 stable at 3 and 12 months. It is noteworthy that the variation of corneal SR or MTF was different 150 from that of ocular and internal optic. No significant differences existed for corneal SR before 151 and after wearing OK lenses, and the corneal MTF decreased significantly after wearing OK 152 lenses.

153 The assessment of MTF at different spatial frequencies is important for estimating the 154 visual quality at different contrast levels. As illustrated in Figs. 1, 2 and 3, the evolution of 155 ocular, corneal and internal MTFs were addressed respectively. The ocular and internal optic 156 MTFs after wearing OK lenses were higher than those before wearing OK lenses at the same 157 spatial frequency. Moreover, the ocular, corneal and internal optic MTFs after wearing OK 158 lenses all decreased with the increase of the spatial frequency, which was similar with the 159 evolution tendency of MTFs before wearing OK lenses.

160

161 162

3.4. Comparison of wavefront aberrations before and after wearing OK lenses

\subsubsection{Comparison of ocular aberrations}

The statistical results of ocular aberrations were listed in Table 4. One can see that the total higher order aberration (HOA), spherical, coma and trefoil aberrations increased obviously after wearing $\mathrm{OK}$ lenses, except the trefoil aberration at 12 months which had no statistical difference when compared with the baseline value. The total aberration, total lower order aberration (LOA) 
168 and defocus aberrations decreased dramatically after wearing OK lenses. One exception was the

169 astigmatism aberration that had no obvious difference after wearing OK lenses.

170

171 3.4.2. Comparison of corneal aberrations

172 As summarized in Table 5, most of the corneal aberrations increased significantly after 173 wearing OK lenses. The exception was the post-treatment astigmatisms and the trefoil aberration 174 at 12 months, which revealed limited difference when compared with the baseline value.

175

176

\subsubsection{Comparison of internal optic aberrations}

177

As shown in Table 6 that the total aberration, total LOA and defocus aberrations of internal

178 optic decreased significantly, and the total HOA, coma and trefoil aberrations increased 179 obviously after wearing OK lenses except the trefoil aberration at 12 months. It's remarkable that

180 there were no significant differences for the astigmatism and spherical aberrations after wearing 181 OK lenses when compared with the baseline value.

182

183

\subsubsection{Comparison of CF and CF\% of aberrations}

184

The CF of total aberration, total LOA, total HOA and coma aberrations increased 185 significantly at 12 months, and the corresponding CF\% also increased after wearing OK lenses. 186 In contrast, there were no statistical significances for the CFs of defocus, astigmatism, spherical and trefoil aberrations when compared with that before wearing OK lenses (as summarized in 188 Table 7).

189

190

\section{Discussion}

191 It is well known that orthokeratology is effective for the correction of refractive error [17, 18].

192 In this work, the effectiveness was further verified by the increase of the vision of LogMAR, 193 which relied on the reshaping of cornea that is represented by the variation of the corneal 
194 curvature as well as the Q-value and e-value. Moreover, the corneal curvature, Q-value and e-

195 value remained unchanged for months after wearing OK lenses, which demonstrated the stable 196 and long-term correction effect of OK.

197 However, the improvement or decline of visual quality after wearing OK lenses still remains 198 in dispute. The eye is a complex and precise refractive system, and there are numbers of factors 199 that affect the visual quality, e.g. the diffraction, scattering and refraction of light. Defined by the 200 deviation between the actual and theoretical wavefront, wavefront aberration is known to reflect 201 the influence of optical factors on the visual imaging. In this work, the objective assessment 202 parameters of vision quality and wavefront aberrations were systematically measured and 203 analyzed respectively based on 3 sections of cornea, ocular and internal optic. The purpose of the 204 individual evaluation was to explore whether there were any differences and interactions 205 between different parts of the optical system. Similar researches have been seldom reported in 206 the published literature to the authors' knowledge.

207 Generally, the variations of ocular SR and MTF indicated that wearing OK lenses could help 208 ameliorate the visual quality of the juvenile myopias, and the visual quality in different spacial 209 frequencies was also improved. Individually, the decrease of corneal SR and MTF indicated the 210 negative effect of $\mathrm{OK}$ associated with corneal reshaping. The small irregularity of cornea after 211 reshaping can increase the light path of different parts of pupil, and thus produce higher corneal 212 aberrations. And the aberrations of the ocular and internal optic changed differently from the 213 corneal aberrations after wearing OK lenses. The various evolutions of wavefront aberrations 214 demonstrated that the compensating effect worked in this phenomenon, which has been noticed 215 in previous works [19-21]. The increased CF and CF\% of some wavefront aberrations also 216 supported this view. The high corneal aberration can be compensated by the intraocular effect, 217 resulting in smaller aberration of the whole eye than that of the cornea. Moreover, the increase of 218 internal optic SR and MTF might cover the decrease of corneal SR and MTF, which finally 219 results in the increase of ocular SR and MTF and the improvement of visual quality.

220 However, the corneal astigmatism did not increase after wearing OK lenses. It was 221 speculated that the stable corneal astigmatism was related to the uniformity of the remodeling 222 force caused by the OK lens because the corneal curvature decreased synchronously along the 223 vertical axis and horizontal axis. The compensating effect of internal optic astigmatism on the 224 corneal astigmatism depended on the natural lens, and was related to the contraction of the 
225 ciliary muscles in all directions. As the corneal astigmatism did not increase, there was no need

226 to activate the internal optic astigmatism to act as a neutralizing role. Therefore, both the ocular

227 and internal optic astigmatism did not increase. It also indicated that the compensation

228 mechanism was a kind of dynamic compensation. The other two noticeable features were that the

229 spherical aberration of internal optic remained unchanged after wearing OK lenses, and the

230 trefoil aberrations of the ocular, internal optic and cornea at 12 months all decreased to the

231 baseline value. It has been pointed out that the combination of trefoil, spherical aberration and

232 vertical coma aberration might help promote the growth of the eyeballs [22]. Moreover, the

233 vertical trefoil coupled with vertical coma could maximize the retinal image quality and reduce

234 the deleterious impact of trefoil in visual performance [23]. However, it is still not clear for the

235 comprehension of the connection between the distinctive changes of spherical and trefoil

236 aberration with the variation of eyeball and the visual quality, which deserves further exploration.

237

238 Furthermore, the compensation mechanisms are not clear so far [24, 25]. One possible 239 explanation might be the adaptive response of eye accommodation to corneal remolding, which 240 primarily relies on the contribution of lens. The OK lens can reduce the hypermetropic refraction 241 of the peripheral retina and improve the sensitivity and accuracy of accommodation that has tight 242 connection with the intraocular lens and ciliary muscle. With age, light, surgery or eye disease, 243 this compensation mechanism may attenuate or even be broken, which might help comprehend 244 the few inconformity of visual quality in some researches.

245 Last but not the least, it should be noted that there still remain some limitations in the present 246 work, e.g. some complications, including the lens decentration, dry eyes and corneal epithelium 247 damage, were not well addressed. A potential selection bias was also introduced by the strict 248 exclusion criteria about the adverse complications of wearing orthokeratology lenses. In addition, 249 one can also try to increase the sample size and follow-up time to further verify the statistical 250 results as observed in this work.

251

\section{5. Conclusion}

253 In conclusion, orthokeratology is effective for the correction of refractive error and 254 improvement of vision quality of juvenile myopia over the one-year follow-up period. 


\section{References}

257 [1] P. Cho, S.W. Cheung, J. Mountford, P. White, Good clinical practice in orthokeratology, 258 Contact lens \& anterior eye : the journal of the British Contact Lens Association 31(1) 259 (2008) 17-28.

260 [2] T. Dave, D. Ruston, Current trends in modern orthokeratology, Ophthalmic \& 261 physiological optics : the journal of the British College of Ophthalmic Opticians 262 (Optometrists) 18(2) (1998) 224-33.

263 [3] Y. Lian, M. Shen, S. Huang, Y. Yuan, Y. Wang, D. Zhu, J. Jiang, X. Mao, J. Wang, F. Lu, 264 Corneal Reshaping and Wavefront Aberrations During Overnight Orthokeratology, Eye \& 265 Contact Lens-Science and Clinical Practice 40(3) (2014) 161-168.

266 [4] A. Queiros, C. Villa-Collar, A. Ramon Gutierrez, J. Jorge, M.S. Ribeiro-Queiros, S.C. 267 Peixoto-de-Matos, J. Manuel Gonzalez-Meijome, Anterior and Posterior Corneal Elevation 268 After Orthokeratology and Standard and Customized LASIK Surgery, Eye \& Contact Lens269 Science and Clinical Practice 37(6) (2011) 354-358.

270 [5] A. Queiros, J.M. Gonzalez-Meijome, C. Villa-Collar, A. Ramon Gutierrez, J. Jorge, Local 271 Steepening in Peripheral Corneal Curvature After Corneal Refractive Therapy and LASIK, 272 Optometry and Vision Science 87(6) (2010) 432-439.

273 [6] J.J. Walline, L.A. Jones, L.T. Sinnott, Corneal reshaping and myopia progression, British 274 Journal of Ophthalmology 93(9) (2009) 1181-1185.

275 [7] P. Cho, S.-W. Cheung, Retardation of Myopia in Orthokeratology (ROMIO) Study: A 2276 Year Randomized Clinical Trial, Investigative Ophthalmology \& Visual Science 53(11) 277 (2012) 7077-7085.

278 [8] Y. Kobayashi, R. Yanai, N. Chikamoto, T.-i. Chikama, K. Ueda, T. Nishida, Reversibility of 279 effects of orthokeratology on visual acuity, refractive error, corneal topography, and 280 contrast sensitivity, Eye \& Contact Lens-Science and Clinical Practice 34(4) (2008) 224281228.

282 [9] D. Chen, A.K.C. Lam, P. Cho, Posterior corneal curvature change and recovery after 6 283 months of overnight orthokeratology treatment, Ophthalmic and Physiological Optics $28430(3)$ (2010) 274-280.

285 [10] E. Santolaria, A. Cervino, A. Queiros, R. Brautaset, J.M. Gonzalez-Meijome, Subjective 286 Satisfaction in Long-term Orthokeratology Patients, Eye \& Contact Lens-Science and 287 Clinical Practice 39(6) (2013) 388-393.

288 [11] C. C.-M., H. Zhong, L.-B. Cheng, P.-B. Wu, X. Wang, Y. Zhou, T.-H. Huang, Clinical 289 research on overnight orthokeratology for vision quality, International Eye Science 15(12) 290 (2015) 2127-2129.

291 [12] W. Ma, M. Liao, H.-Z. Jin, L.-Q. Liu, Evaluation of visual quality after overnight 292 orthokeratology in pre-adolescent myopes, Zhonghua Shiyan Yanke Zazhi/Chinese Journal 293 of Experimental Ophthalmology 30(12) (2012) 1104-1109.

294 [13] J. Li, C. Yang, W. Xie, G. Zhang, X. Li, S. Wang, X. Yang, J. Zeng, Predictive role of corneal 295 Q-value differences between nasal-temporal and superior-inferior quadrants in 296 orthokeratology lens decentration, Medicine 96(2) (2017). 
297 [14] M. Kaido, Y. Matsumoto, Y. Shigeno, R. Ishida, M. Dogru, K. Tsubota, Corneal 298 Fluorescein Staining Correlates with Visual Function in Dry Eye Patients, Investigative 299 Ophthalmology \& Visual Science 52(13) (2011) 9516-9522.

300 [15] D.M. Win-Hall, A. Glasser, Objective accommodation measurements in pseudophakic 301 subjects using an autorefractor and an aberrometer, Journal of Cataract and Refractive 302 Surgery 35(2) (2009) 282-290.

303 [16] X. Chen, Y. Lu, J.-h. Dai, L. Wang, Agreement measurement of ocular wavefront 304 aberrations with three different aberrometers, [Zhonghua yan ke za zhi] Chinese journal of 305 ophthalmology 45(4) (2009) 332-7.

306 [17] Y. Zhong, Z. Chen, F. Xue, H. Miao, X. Zhou, Central and Peripheral Corneal Power 307 Change in Myopic Orthokeratology and Its Relationship With 2-Year Axial Length Change, 308 Investigative Ophthalmology \& Visual Science 56(8) (2015) 4514-4519.

309 [18] T. Hiraoka, C. Okamoto, Y. Ishii, T. Kakita, T. Oshika, Contrast sensitivity function and 310 ocular higher-order aberrations following overnight orthokeratology, Investigative 311 Ophthalmology \& Visual Science 48(2) (2007) 550-556.

[19] J.E. Kelly, T. Mihashi, H.C. Howland, Compensation of corneal horizontal/vertical astigmatism, lateral coma, and spherical aberration by internal optics of the eye, Journal of Vision 4(4) (2004) 262-271.

[20] P. Artal, A. Guirao, E. Berrio, D.R. Williams, Compensation of corneal aberrations by the internal optics in the human eye, Journal of Vision 1(1) (2001).

[21] D. Gatinel, P.-A. Adam, S. Chaabouni, J. Munck, M. Thevenot, T. Hoang-Xuan, S. Pieger, M. Fujieda, H.S. Bains, Comparison of Corneal and Total Ocular Aberrations Before and After Myopic LASIK, Journal of Refractive Surgery 26(5) (2010) 333-340.

[22] T. Buehren, D.R. Iskander, M.J. Collins, B. Davis, Potential higher-order aberration cues for sphero-cylindrical refractive error development, Optometry and Vision Science 84(3) (2007) 163-174.

[23] E.A. Villegas, E. Alcon, P. Artal, Prevalence of the Trefoil Aberration Term and Its Visual Impact, Investigative Ophthalmology \& Visual Science 48 (2007) 1509.

[24] Q. Chen, M. Li, Y. Yuan, R. Me, Y. Yu, G. Shi, B. Ke, Interaction between Corneal and Internal Ocular Aberrations Induced by Orthokeratology and Its Influential Factors, Biomed Research International (2017).

[25] E. Berrio, J. Tabernero, P. Artal, Optical aberrations and alignment of the eye with age, Journal of Vision 10(14) (2010). 
Figure 1

The evolution of ocular MTF at different spatial frequencies before and at $1,3,12$ months after wearing the OK lens. Error bar: standard devision.

Change of ocular MTF under different spatial frequencies

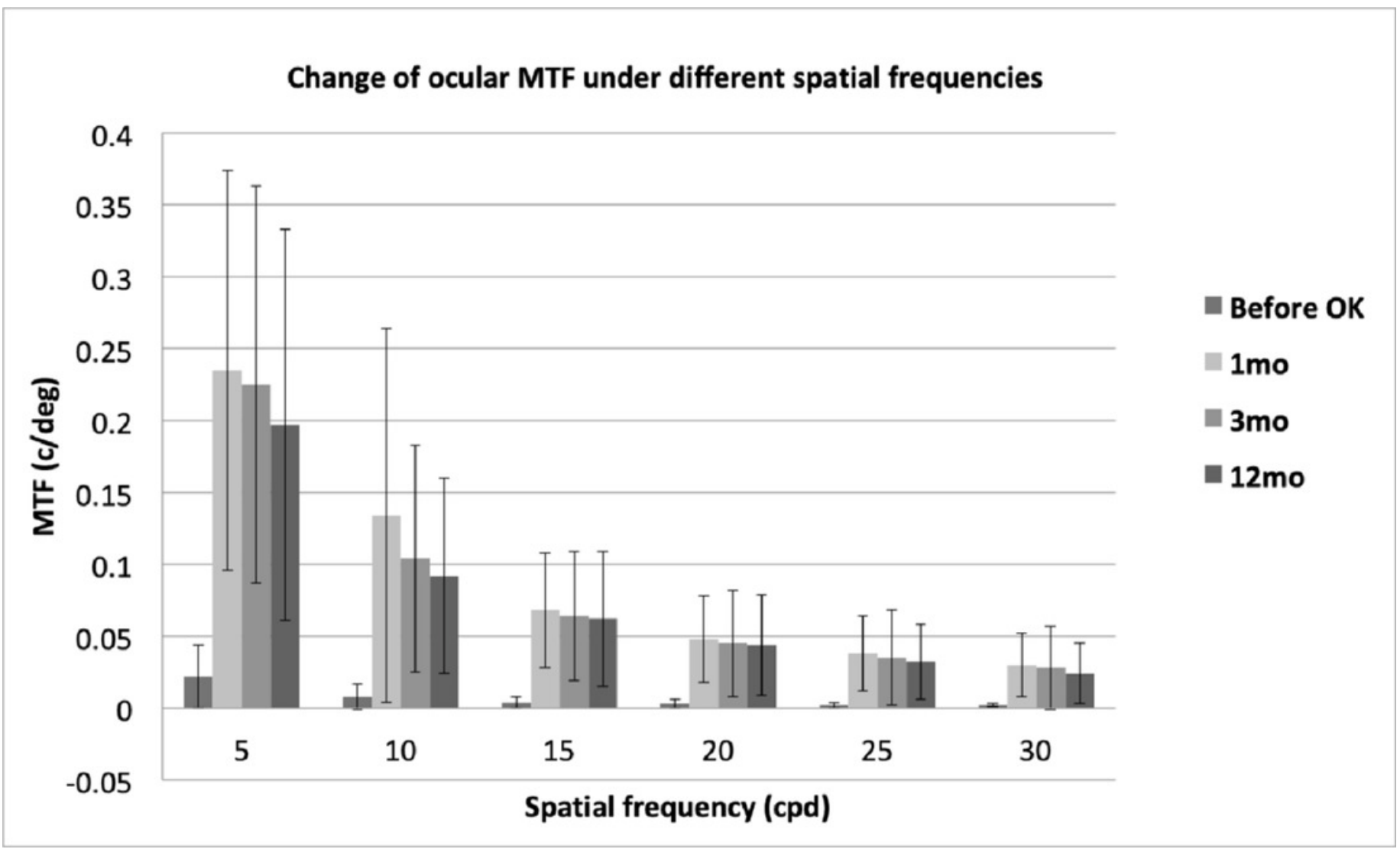


Figure 2

The evolution of corneal MTF at different spatial frequencies before and at 1, 3, 12 months after wearing OK lens. Error bar: standard devision.

Change of corneal MTF under different spatial frequencies

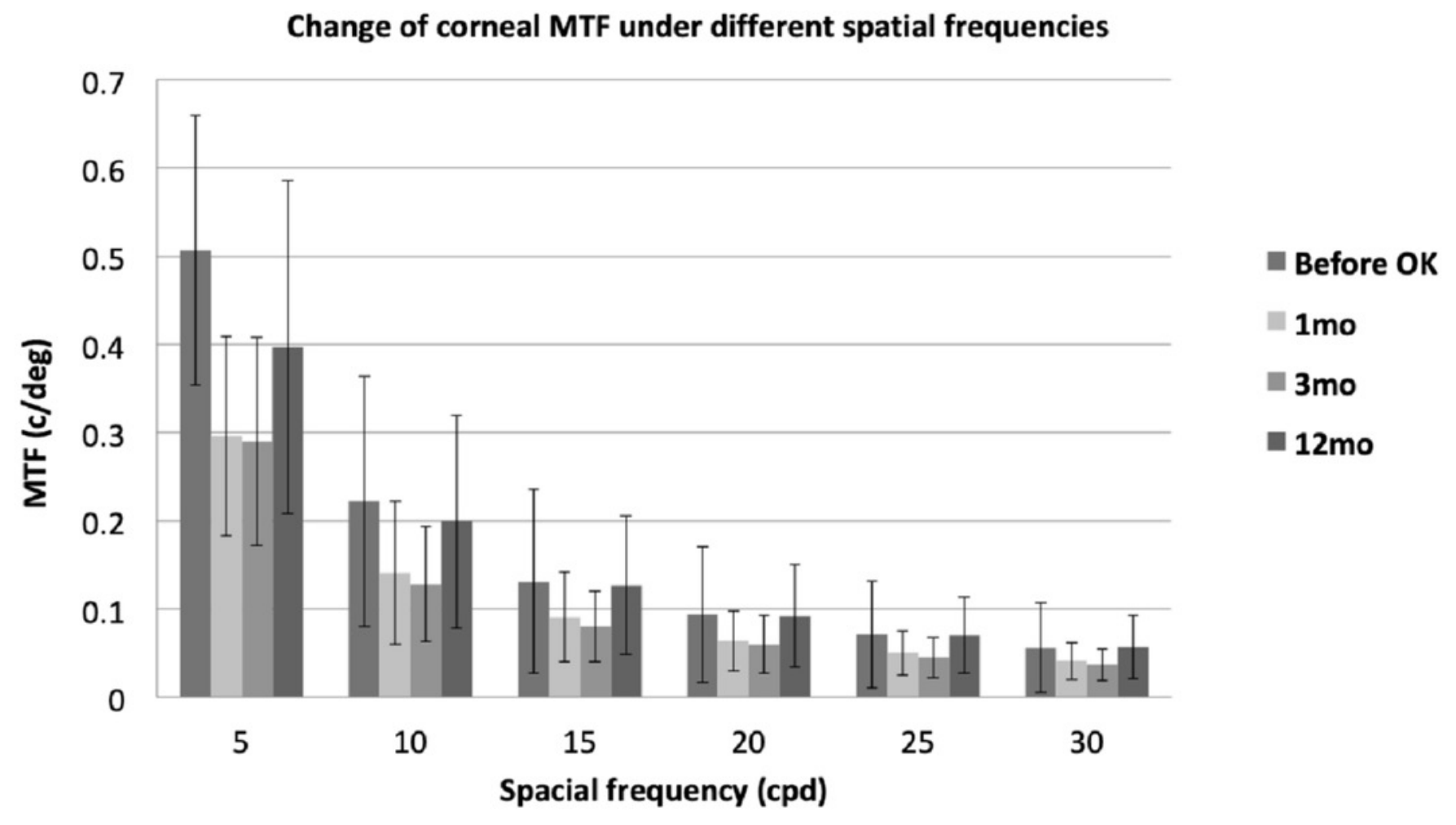


Figure 3

The evolution of internal optic MTF at different spatial frequencies before and at 1, 3, 12 months after wearing OK lens. Error bar: standard devision.

Change of internal optic MTF under different spatial frequencies

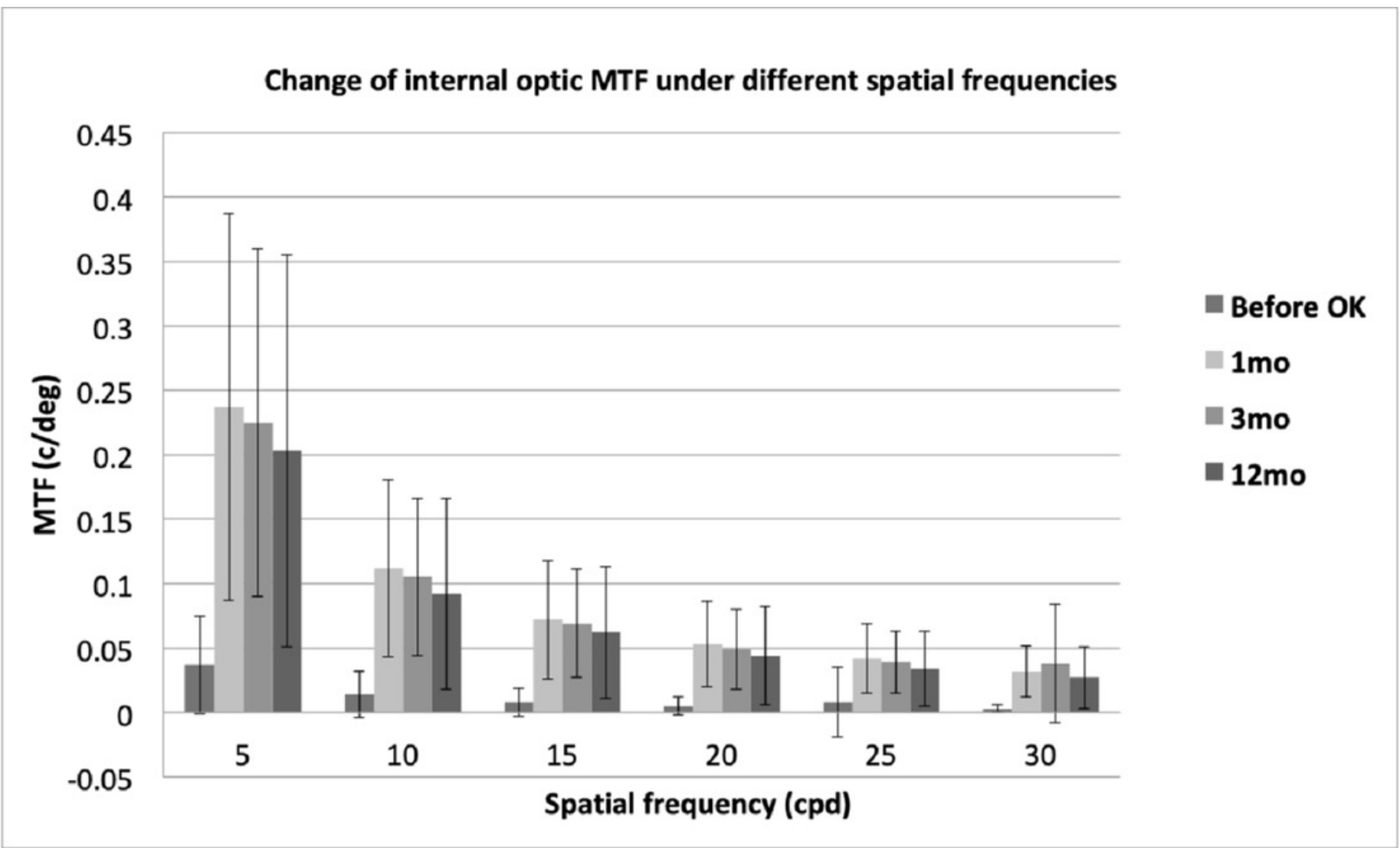




\section{Table $\mathbf{1}$ (on next page)}

Descriptive statistics (mean $\pm \mathrm{SE}$ ) of myopic teenagers

* 4 eyes had astigmatism above -1.00D. 
Table 1. Descriptive statistics (mean $\pm \mathrm{SE}$ ) of myopic teenagers

\begin{tabular}{|c|c|c|c|}
\hline & Before OK & 12 months after OK & $p$ \\
\hline Gender & 19 female, 17 male & l & \\
\hline Age (years) & $10.8 \pm 2.2$ & l & \\
\hline Sphere diopter（D） & $-3.403 \pm 1.580$ & $-0.410 \pm 0.330$ & $<0.001$ \\
\hline Cylinder diopter (D) & $-0.465 \pm 0.539^{*}$ & $-0.210 \pm 0.210$ & 0.008 \\
\hline LogMAR & $-0.788 \pm 0.242$ & $-0.008 \pm 0.177$ & $<0.001$ \\
\hline
\end{tabular}

1 Table 1: * 4 eyes had astigmatism above $-1.00 \mathrm{D}$.

2 


\section{Table 2 (on next page)}

Comparison of corneal curvature, Q-value and e-value

The corneal curvature decreased significantly after wearing OK lens. Q-value increased at 1 month and decreased dramatically after 3 months. There were no significant differences for e-value before and after wearing OK lens. Data is expressed as mean \pm SD. * Compared with that before $\mathrm{OK}, p>$ adjusted alpha. 
Table 2. Comparison of corneal curvature, Q-value and e-value

\begin{tabular}{llllll}
\hline & Before OK & 1 month & 3 months & 12 months & $p$ \\
\hline $\begin{array}{l}\text { Horizontal corneal } \\
\text { curvature, D }\end{array}$ & $41.065 \pm 1.781$ & $40.273 \pm 1.420$ & $40.384 \pm 1.336$ & $40.258 \pm 1.357$ & $<0.001$ \\
Vertical corneal & $43.354 \pm 2.075$ & $41.406 \pm 1.419$ & $41.219 \pm 1.942$ & $41.320 \pm 1.859$ & $<0.001$ \\
curvature, D & & & & & \\
Q-value & $-0.203 \pm 0.186$ & $0.375 \pm 0.643$ & $0.224 \pm 0.523$ & $0.202 \pm 0.532$ & $<0.001$ \\
& & & & & \\
e-value & $0.482 \pm 0.129$ & $0.528 \pm 0.211^{*}$ & $0.480 \pm 0.180^{*}$ & $0.424 \pm 0.210^{*}$ & 0.397 \\
\hline
\end{tabular}

1 Table 2: The corneal curvature decreased significantly after wearing OK lens. Q-value increased at 1 month and decreased

2 dramatically after 3 months. There were no significant differences for e-value before and after wearing OK lens. Data is 3 expressed as mean $\pm \mathrm{SD} .{ }^{*}$ Compared with that before $\mathrm{OK}, p>$ adjusted alpha.

\section{4}




\section{Table 3 (on next page)}

Comparison of SR and MTF

There were no significant differences of corneal SR before and after wearing OK lens, and the corneal MTF decreased after wearing OK lens significantly. After wearing OK lens the ocular and internal optic SR and MTF increased obviously. Data is expressed as mean \pm SD. *Compared with that before OK, $p>$ adjusted alpha. 
Table 3 Comparison of SR and MTF

\begin{tabular}{cllllc}
\hline & Before OK & 1 month & 3 months & 12 months & $p$ \\
\hline Ocular SR & $0.001 \pm 0.001$ & $0.017 \pm 0.019$ & $0.028 \pm 0.033$ & $0.017 \pm 0.026$ & $<0.001$ \\
Corneal SR & $0.051 \pm 0.048$ & $0.027 \pm 0.020^{*}$ & $0.028 \pm 0.026^{*}$ & $0.031 \pm 0.023^{*}$ & 0.237 \\
Internal optic & & & & & \\
SR & $0.001 \pm 0.002$ & $0.014 \pm 0.012$ & $0.020 \pm 0.020$ & $0.020 \pm 0.026$ & $<0.001$ \\
$\begin{array}{c}\text { Ocular MTF, } \\
\text { c/deg }\end{array}$ & $0.054 \pm 0.018$ & $0.141 \pm 0.069$ & $0.165 \pm 0.083$ & $0.138 \pm 0.074$ & $<0.001$ \\
$\begin{array}{c}\text { Corneal MTF, } \\
\text { c/deg }\end{array}$ & $0.265 \pm 0.091$ & $0.189 \pm 0.068$ & $0.184 \pm 0.052$ & $0.205 \pm 0.072$ & 0.001 \\
$\begin{array}{c}\text { Internal optic } \\
\text { MTF, c/deg }\end{array}$ & $0.062 \pm 0.026$ & $0.137 \pm 0.056$ & $0.165 \pm 0.081$ & $0.148 \pm 0.089$ & $<0.001$ \\
\hline
\end{tabular}

1 Table 3: There were no significant differences of corneal SR before and after wearing OK lens, and the corneal MTF decreased

2 after wearing OK lens significantly. After wearing OK lens the ocular and internal optic SR and MTF increased obviously. Data

3 is expressed as mean $\pm \mathrm{SD}$. *Compared with that before $\mathrm{OK}, p>$ adjusted alpha.

4 


\section{Table 4 (on next page)}

Comparison of ocular aberrations

After wearing OK lens the total HOA, spherical, coma and trefoil aberrations increased, and the total aberration, total LOA and defocus aberration decreased. Data is expressed as mean \pm SD. * Compared to that before OK, $p>$ adjusted alpha. 
1

Table 4 Comparison of ocular aberrations

\begin{tabular}{llllll}
\hline & Before OK & 1 month & 3 months & 12 months & $p$ \\
\hline Total & $2.446 \pm 1.055$ & $1.518 \pm 1.038$ & $1.564 \pm 1.164$ & $1.741 \pm 1.326$ & $<0.001$ \\
aberration & & & & & \\
Total LOA & $2.441 \pm 1.054$ & $1.371 \pm 1.012$ & $1.422 \pm 1.126$ & $1.628 \pm 1.285$ & $<0.001$ \\
Defocus & $2.404 \pm 1.029$ & $1.248 \pm 1.051$ & $1.328 \pm 1.182$ & $1.445 \pm 1.252$ & $<0.001$ \\
Astigmatism & $0.262 \pm 0.119$ & $0.392 \pm 0.227^{*}$ & $0.364 \pm 0.215^{*}$ & $0.372 \pm 0.157^{*}$ & 0.017 \\
Total HOA & $0.141 \pm 0.068$ & $0.496 \pm 0.488$ & $0.511 \pm 0.328$ & $0.497 \pm 0.492$ & $<0.001$ \\
Spherical & $0.032 \pm 0.037$ & $0.180 \pm 0.191$ & $0.189 \pm 0.145$ & $0.194 \pm 0.182$ & $<0.001$ \\
Coma & $0.075 \pm 0.052$ & $0.347 \pm 0.253$ & $0.358 \pm 0.283$ & $0.246 \pm 0.144$ & $<0.001$ \\
Trefoil & $0.049 \pm 0.026$ & $0.109 \pm 0.092$ & $0.137 \pm 0.091$ & $0.108 \pm 0.084^{*}$ & $<0.001$ \\
\hline
\end{tabular}

2 Table 4: After wearing OK lens the total HOA, spherical, coma and trefoil aberrations increased, and the total aberration, total

3 LOA and defocus aberration decreased. Data is expressed as mean \pm SD. * Compared to that before OK, $p>$ adjusted alpha. 4 


\section{Table 5 (on next page)}

Comparison of corneal aberrations ations

The corneal aberrations increased significantly after wearing OK lens except astigmatism. Data is expressed as mean \pm SD. * Compared to that before $O K, p>$ adjusted alpha. 
Table 5 Comparison of corneal aberrations

\begin{tabular}{llllll}
\hline & Before OK & 1 month & 3 months & 12 months & $p$ \\
\hline $\begin{array}{l}\text { Total } \\
\text { aberration }\end{array}$ & $0.544 \pm 0.213$ & $1.187 \pm 0.980$ & $1.199 \pm 0.601$ & $1.372 \pm 2.038$ & $<0.001$ \\
$\begin{array}{l}\text { Total LOA } \\
\text { Defocus }\end{array}$ & $0.529 \pm 0.222$ & $1.012 \pm 0.754$ & $1.050 \pm 0.531$ & $1.147 \pm 1.650$ & $<0.001$ \\
Astigmatism & $0.131 \pm 0.125$ & $0.670 \pm 0.699$ & $0.790 \pm 0.600$ & $0.865 \pm 1.360$ & $<0.001$ \\
Total HOA & $0.108 \pm 0.037$ & $0.587 \pm 0.657$ & $0.544 \pm 0.346$ & $0.717 \pm 1.220$ & $<.040$ \\
Spherical & $0.033 \pm 0.026$ & $0.151 \pm 0.122$ & $0.175 \pm 0.114$ & $0.211 \pm 0.376$ & $<0.001$ \\
Coma & $0.043 \pm 0.031$ & $0.435 \pm 0.349$ & $0.487 \pm 0.323$ & $0.414 \pm 0.324$ & $<0.001$ \\
Trefoil & $0.058 \pm 0.029$ & $0.116 \pm 0.070$ & $0.134 \pm 0.076$ & $0.111 \pm 0.075^{*}$ & $<0.001$ \\
\hline
\end{tabular}

1 Table 5: The corneal aberrations increased significantly after wearing OK lens except astigmatism. Data is expressed as 2 mean \pm SD. * Compared to that before $\mathrm{OK}, p>$ adjusted alpha.

3 


\section{Table 6(on next page)}

Comparison of internal optic aberrations

The total aberration, total LOAs and defocus aberration decreased significantly after wearing OK lens, and the total HOAs, spherical, coma and trefoil aberration increased significantly. Data is expressed as mean \pm SD. * Compared to that before OK, $p>$ adjusted alpha. 
Table 6 Comparison of internal optic aberrations

\begin{tabular}{llllll}
\hline & Before OK & 1 month & 3 months & 12 months & $p$ \\
\hline $\begin{array}{l}\text { Total } \\
\text { aberration }\end{array}$ & $2.287 \pm 0.997$ & $1.287 \pm 0.854$ & $1.201 \pm 0.869$ & $1.528 \pm 1.457$ & $<0.001$ \\
$\begin{array}{l}\text { Total LOA } \\
\text { Defocus }\end{array}$ & $2.309 \pm 1.029$ & $1.150 \pm 0.874$ & $1.087 \pm 0.894$ & $1.278 \pm 1.132$ & $<0.001$ \\
Astigmatism & $0.320 \pm 0.123$ & $0.426 \pm 0.253^{*}$ & $0.436 \pm 0.213^{*}$ & $0.331 \pm 0.174^{*}$ & 0.008 \\
Total HOA & $0.154 \pm 0.054$ & $0.447 \pm 0.321$ & $0.413 \pm 0.219$ & $0.622 \pm 1.080$ & $<0.001$ \\
Spherical & $-0.001 \pm 0.037$ & $0.030 \pm 0.136$ & $0.013 \pm 0.092$ & $-0.017 \pm 0.442$ & 0.519 \\
Coma & $0.086 \pm 0.049$ & $0.286 \pm 0.159$ & $0.291 \pm 0.193$ & $0.319 \pm 0.239$ & $<0.001$ \\
Trefoil & $0.074 \pm 0.026$ & $0.166 \pm 0.118$ & $0.148 \pm 0.092$ & $0.112 \pm 0.073 *$ & $<0.001$ \\
\hline
\end{tabular}

1 Table 6: The total aberration, total LOAs and defocus aberration decreased significantly after wearing OK lens, and the total

2 HOAs, spherical, coma and trefoil aberration increased significantly. Data is expressed as mean \pm SD. * Compared to that before 3 OK, $p>$ adjusted alpha. 


\section{Table 7 (on next page)}

Comparison of compensating factors and compensation rates for aberrations

The CF and CF\% of total aberration, total LOA, total HOA and coma aberrations increased at 12 months. Data is expressed as mean \pm SD. 
1

Table 7 Comparison of compensating factors and compensation rates for

\begin{tabular}{|c|c|c|c|c|c|}
\hline & \multicolumn{2}{|c|}{$\mathrm{CF}$} & \multirow{2}{*}{$p$} & \multicolumn{2}{|c|}{$\mathrm{CF} \%$} \\
\hline & Before OK & 12 months & & Before OK & 12 months \\
\hline $\begin{array}{l}\text { Total } \\
\text { aberration }\end{array}$ & $-4.013 \pm 2.532$ & $-1.064 \pm 1.788$ & $<0.001$ & $0 \%$ & $36.1 \%$ \\
\hline Total LOA & $-4.407 \pm 3.022$ & $-1.390 \pm 2.155$ & $<0.001$ & $0 \%$ & $30.6 \%$ \\
\hline Defocus & $-31.646 \pm 54.817$ & $-32.438 \pm 166.855$ & 0.979 & $0 \%$ & $36.1 \%$ \\
\hline Astigmatism & $-0.329 \pm 3.271$ & $-0.385 \pm 0.970$ & 0.921 & $86.1 \%$ & $38.9 \%$ \\
\hline Total HOA & $-0.379 \pm 0.719$ & $0.082 \pm 0.435$ & 0.001 & $33.3 \%$ & $61.1 \%$ \\
\hline Spherical & $-0.679 \pm 3.793$ & $-1.621 \pm 4.460$ & 0.356 & $58.3 \%$ & $36.1 \%$ \\
\hline Coma & $-1.894 \pm 2.538$ & $0.226 \pm 0.542$ & $<0.001$ & $19.4 \%$ & $66.7 \%$ \\
\hline Trefoil & $-0.177 \pm 0.974$ & $-0.142 \pm 1.019$ & 0.888 & $63.9 \%$ & $63.9 \%$ \\
\hline
\end{tabular}

2 Table 7: The CF and CF\% of total aberration, total LOA, total HOA and coma aberrations increased at 12 months. Data is 3 expressed as mean \pm SD.

4 